\title{
ACTIVE VIBRATION SUPPRESSION R+D FOR THE NEXT LINEAR COLLIDER*
}

\author{
S. Allison, L. Eriksson, J. Frisch, L. Hendrickson, T. Himel, K. Luchini, A. Seryi, Stanford Linear \\ Accelerator Center, Menlo Park CA 94305, USA
}

\begin{abstract}
The nanometer scale beam sizes at the interaction point in linear colliders limit the allowable motion of the final focus magnets. We have constructed a prototype system to investigate the use of active vibration damping to control magnet motion. Inertial sensors are used to measure the position of a test mass, and a DSP based system provides feedback using electrostatic pushers. Simulation and experimental results for the control of a mechanically simple system are presented.
\end{abstract}

\section{REQUIREMENTS}

\subsection{Stability Requirements}

The beam spot sizes at the NLC interaction point have vertical beam sizes of a few nanometers and require relative position stability of $\sim 1 \mathrm{~nm}$ RMS in order to maintain luminosity. A primary contributor to the relative beam motion is the mechanical motion of the final quadrupoles.

The beam-beam deflection from the interaction point provides a direct measurement of the relative positions of the beams. This allows the beam steering to be adjusted in feedback at the $120 \mathrm{~Hz}$ beam rate to attenuate low frequency motions. In addition, as the luminosity is determined by the differential motion of the beams, the correlation of the ground motion reduces the effective beam motion at low frequencies.

The final quadrupoles need to be mounted cantilevered into the physics detector. This mounting is likely to introduce mechanical resonances that will amplify the ground motion at some frequencies. In addition, the ground motion spectrum (temporal and spatial) varies dramatically between sites. See [1] for a discussion of the requirements on position stability for a linear collider final focus.

\subsection{Stabilization Experiment Assumptions}

Due to uncertainties in the location of the accelerator and in beam optics and feedback design, it is not yet possible to specify precise requirements on the stability of the final focus support magnets. Instead we are developing hardware and software to test the capabilities of an active vibration suppression system. In order to evaluate the system performance we are making the following assumptions:

\footnotetext{
- Work Supported by DOE contract DE-AC03-76SF00515
}

1. The ground motion at the existing SLD collider hall is a reasonable and pessimistic model for the NLC collider hall. Initial vibration experiments are being performed in a test lab which has higher vibration that the SLD collider hall. The test apparatus will be moved to the collider hall for final testing.

2. The beam motion at the IP will be dominated by the vibration of the final quadrupoles as these are the most sensitive optical elements in the system. Work is also underway to understand the effects of vibration and feedback in other elements. [2]

\subsection{Overall Experimental Plan}

The vibration stabilization experiment will proceed in several steps. We are nearing completion of stage 1 .

1. A single simple object will be stabilized in all 6 solid body degrees of freedom, using conventional geophones as inertial sensors.

2. Electrostatic inertial sensors capable of operating in a strong magnetic field (the detector solenoid) will be developed to replace the geophones.

3. A complex object with internal degrees of freedom, similar in design to a real final focus magnet support, will be inertially stabilized.

\section{PROTOTYPE SYSTEM}

\subsection{Mechanical Design}

A $42 \mathrm{Kg}$ aluminum block is supported on 6 springs that control its solid body degrees of freedom. A set of 6 inertial sensors is used to measure the motion of the block. An additional sensor mounted on the top of the block is used as an independent position readback. Seven electrostatic actuators are used to control the block motion.

Different springs can be installed to adjust the mode frequencies. The initial experiments have been done with a set of springs providing mode frequencies between 3 and $8 \mathrm{~Hz}$, with the predominantly vertical mode frequency at $5.6 \mathrm{~Hz}$.

Initial experiments are being done using accelerometer sensors with flat (acceleration) frequency response from Dc to $\sim 600 \mathrm{~Hz}$. Their noise level is sufficient to detect nanometer RMS motions at frequencies above $\sim 10 \mathrm{~Hz}$. Accelerometers were chosen for initial testing because of their simple response function. They will be replaced with 
lower noise compact geophones $(4 \mathrm{~Hz}$ resonance) for future tests.

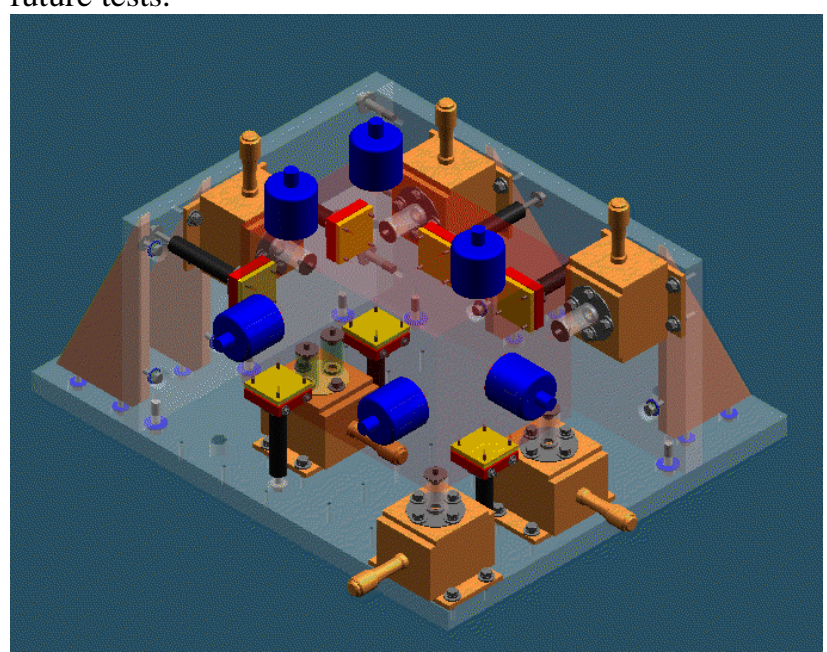

Figure 1: Mechanical System (block removed)

The block motion is controlled with electrostatic actuators: Up to $1 \mathrm{KV}$ is applied across a $\sim 1 \mathrm{~mm}$ gap between $5 \mathrm{~cm}$ plates, producing up to $0.01 \mathrm{~N}$ force, sufficient to control vibration forces transmitted through the support springs. Electrostatic pushers have very low effective stiffness, and faster response speed than piezoelectric actuators.

The force from the actuators is proportional to the square of the applied voltage. This nonlinearity is corrected in software. Note: 7 actuators rather than 6 are used in order to simplify the mechanical design. Two of the actuators are driven together by a single amplifier.

\subsection{Data Acquisition System Design}

The data acquisition and control is performed by a DSP based system in VME format. The DSP is a TMS320C40, operating at $40 \mathrm{MHz}$. Data interface to the 8 input, 8 output, $250 \mathrm{Ks} / \mathrm{s}$ 16-bit analog IO card is though "C 40 " ports which can operate in real-time independently of the VME bus.

Signals from the sensors are amplified with low noise adjustable gain amplifiers. The analog outputs drive high voltage $(1 \mathrm{KV})$ amplifiers that drive the electrostatic pushers.

This system operates the main feedback loop at (typically) 5KHz. Additional sensors, one on the top of the block, and one on the ground are used to monitor the system performance.

\subsection{Feedback System Design}

The feedback algorithm operates on the 6 mechanical normal modes of the block. The sensor signals are converted to modes in a $6 \times 6$ matrix. A single dimensional control filter (with an adjustable number of poles and zeroes) is applied to each measured mode. The output from the filter is converted to required actuator forces in a $6 \times 6$ matrix. Finally the actuator force is converted to the required actuator voltage. (Fig. 2).

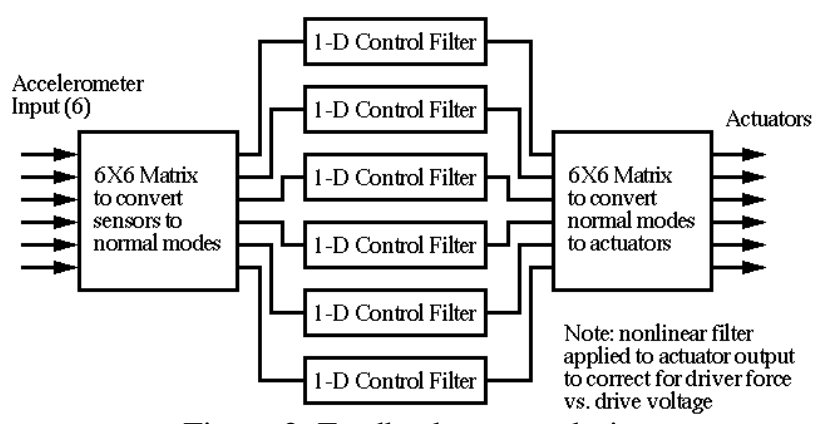

Figure 2: Feedback system design

\subsection{Overall Stabilization Concept}

Low stiffness springs are used to provide low normal mode frequencies: $3-10 \mathrm{~Hz}$. This isolates the block from high frequency noise that would otherwise be difficult to control with feedback.

Without feedback, the high Q mechanical resonances would substantially amplify the ground motion. Feedback based on inertial sensors is used to damp the mechanical resonances. The resulting motion is dominated by low frequencies $(<$ few $\mathrm{Hz})$ where beam based feedback can have large gain (Fig. 3).

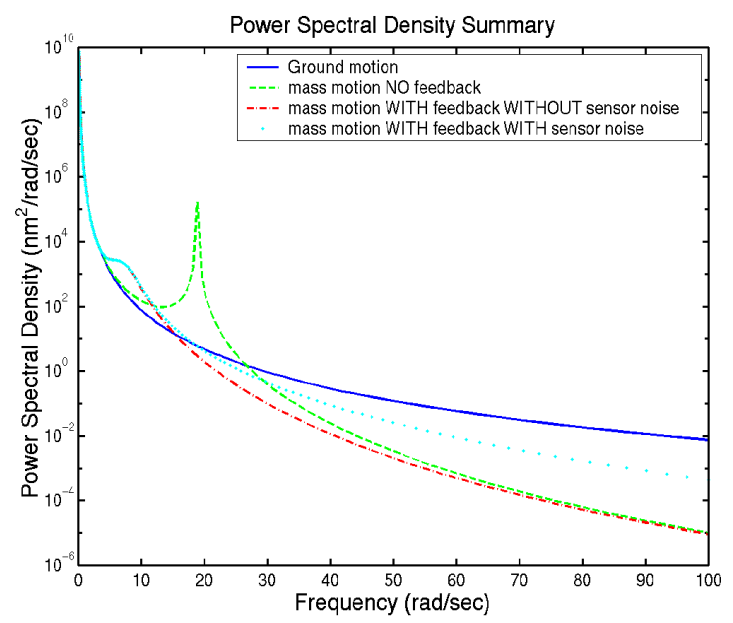

Figure 3: Simulation of feedback

\section{TEST RESULTS}

\subsection{Test Conditions}

The prototype system is being tested in a typical laboratory environment. Significant vibration is generated by (unrelated) equipment containing cooling fans and water and vacuum pumps. After the system software and hardware are fully debugged, it will be moved to the SLD collider hall, which has lower vibration noise and more closely represents a realistic NLC interaction region. Initial testing is also being done with relatively noisy accelerometers. The test system is shown in figure 4 . 


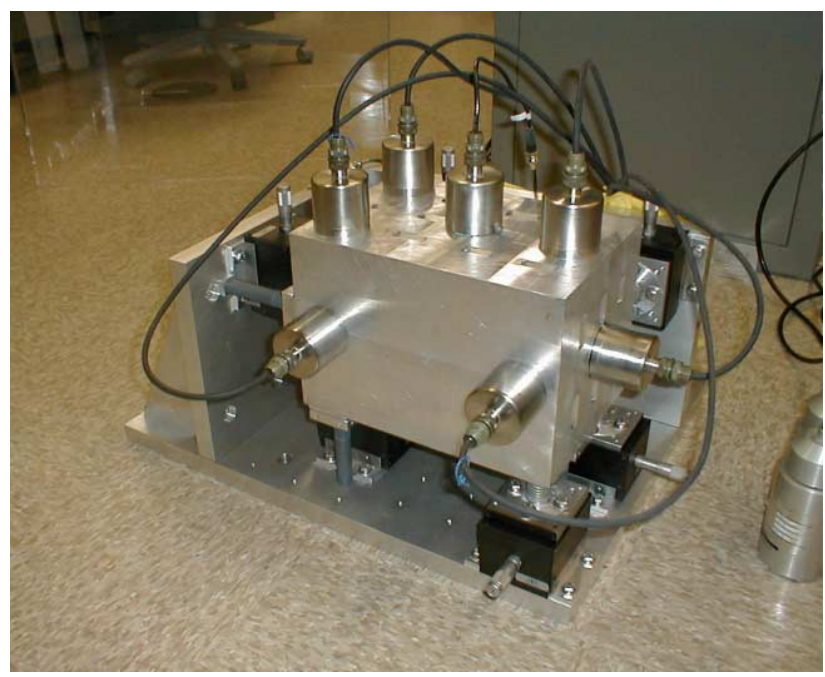

Figure 4: block system (cover removed)

\subsection{Mode Orthogonalization}

The feedback algorithm relies on separately sensing and driving the normal modes of the block. The software needs to identify the mode frequencies and coupling to actuators and sensors.

The approximate mode frequencies are measured by first driving the block with noise in the frequency range of interest. Then the modes are driven at set frequencies near resonance and the precise frequencies and coupling to actuators and sensors is measured. Note that the high mechanical of the modes $(>100)$ allows easy mode identification.

The orthogonalization is tested by driving the linear combination of actuators that couples to one mode, at the resonant frequency of a different mode. If the system is perfectly orthogonalized, no response should be seen by the linear combination of sensors appropriate to the other mode. Note that perfect orthogonalization is not required, as all modes are being damped in feedback. Results of a test are shown in figure 4 .
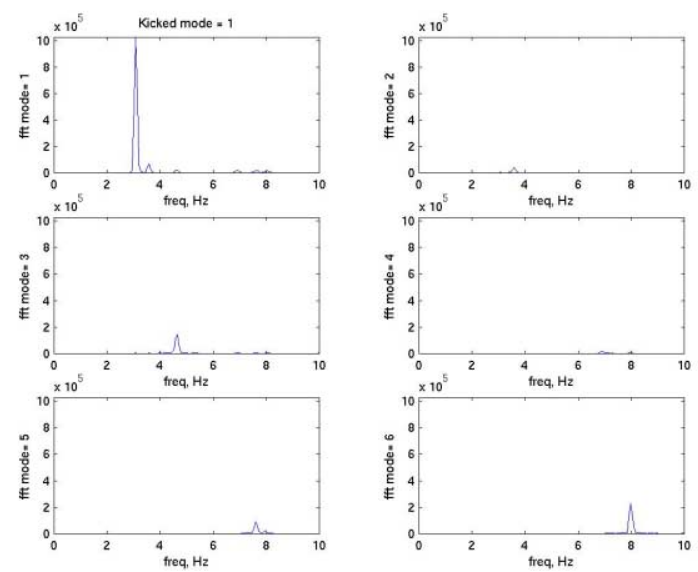

Figure 5: Mode 1 actuator combination driven at other mode frequencies (orthogonalization test)

\subsection{Feedback Performance}

In order to evaluate the performance of the feedback system, a model for a beam-beam deflection feedback was applied to the measured vibration data. It should be noted that without a good model for the beam position noise from other sources, it is difficult to predict the performance of the beam feedback (Fig. 6).

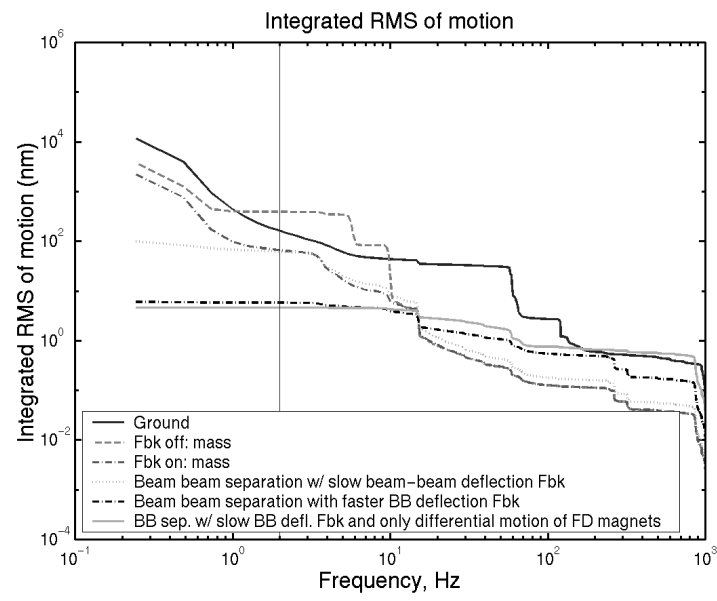

Figure 6: Measured system performance. Simulated effect of beam-beam feedback is shown.

When an assumed electron beam feedback model is applied to the measured motion of the test block in the laboratory, a residual RMS motion of a few nanometers is measured. This is expected to be decreased when the system is moved to a quieter location (SLD hall). In addition, the accelerometers will be replaced with lower noise geophones.

\section{SUMMARY}

A test system to study active vibration control has been constructed and is being tested. Initial testing in a noisy environment and with low sensitivity sensors has shown promising results. Improvements are expected when the lower noise geophone sensors are installed, and the system is moved to the (less noisy) SLD collider hall.

\section{REFERENCES}

1] A. Seryi, "Ground Motion and Vibration Issues for Accelerators" PAC2001, June2001

[2] L. Hendrickson et. al. "NLC Linac Feedback Simulations with Ground Motion", PAC2001, June 2001 